\title{
All in the Stream
}

Benjamin Mba, MD*, Brian P Lucas MD, MS², Nathan Houchens MD³,4, Jennifer Marie Seares, MD1, Udit Joshi, MD¹

This icon represents the patient's case. Each paragraph that follows represents the discussant's thoughts.

'Department of Medicine, John H. Stroger Hospital of Cook County, Chicago, Illinois; ${ }^{2}$ Department of Medicine, White River Junction Veterans Affairs Medical Center, White River Junction, Vermont; ${ }^{3}$ Medicine Service, Veterans Affairs Ann Arbor Healthcare System, Ann Arbor, Michigan; ${ }^{4}$ Department of Internal Medicine, University of Michigan Medical School, Ann Arbor, Michigan.

A 67-year-old man presented to the emergency department with four days of nausea, vomiting, and chills. He was originally from the Philippines but lived in the United States for six years. His past medical history was notable for nephrolithiasis for which a ureteral stent had been placed and was subsequently removed three years prior. He reported no abdominal pain, diarrhea, dysuria, urinary frequency, hematuria, cough, headache, or fever. He was a retired high school teacher and a lifelong nonsmoker.

This patient presents with a nonspecific constellation of constitutional and gastrointestinal (Gl) symptoms. A system-based approach may be helpful when considering the differential diagnosis. Chills most often suggest infection, especially in older patients. With regard to $\mathrm{Gl}$ causes, acute gastroenteritis and other food-borne infections can cause nausea, vomiting, and chills, but these are typically accompanied by abdominal pain and diarrhea and often resolve in less than four days. Abdominal pain would also be expected with cholecystitis as well as more life-threatening causes of nausea such as acute pancreatitis, mesenteric ischemia, and bowel obstruction. In contrast, abdominal pain would not be expected with a central nervous system (CNS) infection such as a brain abscess, which may cause nausea from increased intracranial pressure. Headaches occur in a majority of these cases, making CNS etiologies of nausea less likely. Cardiovascular causes, including myocardial ischemia and infarction, may lead to nausea and vomiting, but these are less likely given the absence of chest pain. Genitourinary causes must be considered, especially given his history of both nephrolithiasis and instrumentation. A stricture or recurrence of nephrolithiasis could lead to acute pyelonephritis or perinephric abscess, although both commonly present with urinary tract symptoms. Uremia, possibly from obstructive uropathy given the patient's history of nephrolithiasis, could also lead to this constellation of symptoms.

On examination, temperature was $101.6^{\circ} \mathrm{F}$, heart rate 126 beats per minute, respiratory rate 18 breaths per

*Corresponding Author: Benjamin Mba, MD, MRCP (UK), FACP; E-mail:

Bmba@cookcountyhhs.org; Telephone: 312-864-7231

Published online first September 18, 2019.

Received: February 4, 2019; Revised: June 25, 2019; Accepted: July 8, 2019

(c) 2019 Society of Hospital Medicine DOI 10.12788/jhm.3286 minute, blood pressure 120/76 $\mathrm{mm} \mathrm{Hg}$, and oxygen saturation $98 \%$ on room air. The oral mucosa was moist, heart sounds were normal without murmurs, lungs were clear to auscultation, and abdomen was soft, nontender, and nondistended. There was no flank tenderness, and penile, testicular, and prostate examination findings were normal.

Laboratory studies revealed a serum sodium of $126 \mathrm{mEq} / \mathrm{L}$, potassium $5.0 \mathrm{mEq} / \mathrm{L}$, chloride $98 \mathrm{mEq} / \mathrm{L}$, bicarbonate 15 $\mathrm{mEq} / \mathrm{L}$, blood urea nitrogen $88 \mathrm{mg} / \mathrm{dL}$, creatinine $9.0 \mathrm{mg} / \mathrm{dL}$, calcium $8 \mathrm{mg} / \mathrm{dL}$, glucose $110 \mathrm{mg} / \mathrm{dL}$, and albumin $3.3 \mathrm{~g} / \mathrm{dL}$. One year prior, serum creatinine was $1.4 \mathrm{mg} / \mathrm{dL}$. His white blood cell (WBC) count was $8.0 \mathrm{k} / \mathrm{uL}$ with normal differential, hemoglobin $11.4 \mathrm{~g} / \mathrm{dL}$ with normal MCV, and platelet count was normal. Serum osmolality was $286 \mathrm{mOsm} / \mathrm{kg}$ and serum parathyroid hormone (PTH) level $63 \mathrm{pg} / \mathrm{mL}$ (normal, 10-65). The urinalysis revealed cloudy urine with a specific gravity 1.009, 54 red blood cells (RBC), 236 WBC, large leukocyte esterase, negative nitrite, trace protein, and no casts or dysmorphic RBCs. A random urine specimen revealed sodium of $86 \mathrm{mEq} / \mathrm{L}$, potassium $16 \mathrm{mEq} / \mathrm{L}$, chloride $80 \mathrm{mEq} / \mathrm{L}$, and creatinine $70 \mathrm{mg} / \mathrm{dL}$.

Fever and tachycardia support an infectious cause of his symptoms. Absent flank tenderness and a normal genitourinary examination have only moderate negative predictive values for acute pyelonephritis and prostatitis, respectively. The most striking laboratory finding is his azotemia. Acute kidney injury (AKI) is more likely than chronic kidney disease (CKD) given that the PTH level is normal and the serum creatinine from a year ago was near normal. The most useful finding to differentiate AKI from CKD is the presence of atrophic kidneys on imaging. The low bicarbonate level indicates a metabolic acidosis. His serum anion gap is $13 \mathrm{mEq} / \mathrm{L}$, which falls above most normal ranges. A mildly elevated serum anion gap together with a "delta serum anion gap/delta serum bicarbonate" ratio less than one suggest concomitant anion gap metabolic acidosis and non anion gap metabolic acidosis. The latter, coupled with a history of nephrolithiasis, may point to the possibility of renal tubular acidosis and AKI caused by urinary tract obstruction. This could also account for the marked hyponatremia. Moreover, his high fractional excretion of sodium (9\%) is not suggestive of prerenal injury, the most common acute renal injury among patients who present to the emergency department. Hematuria carries a broad differential diagnosis, but most com- 


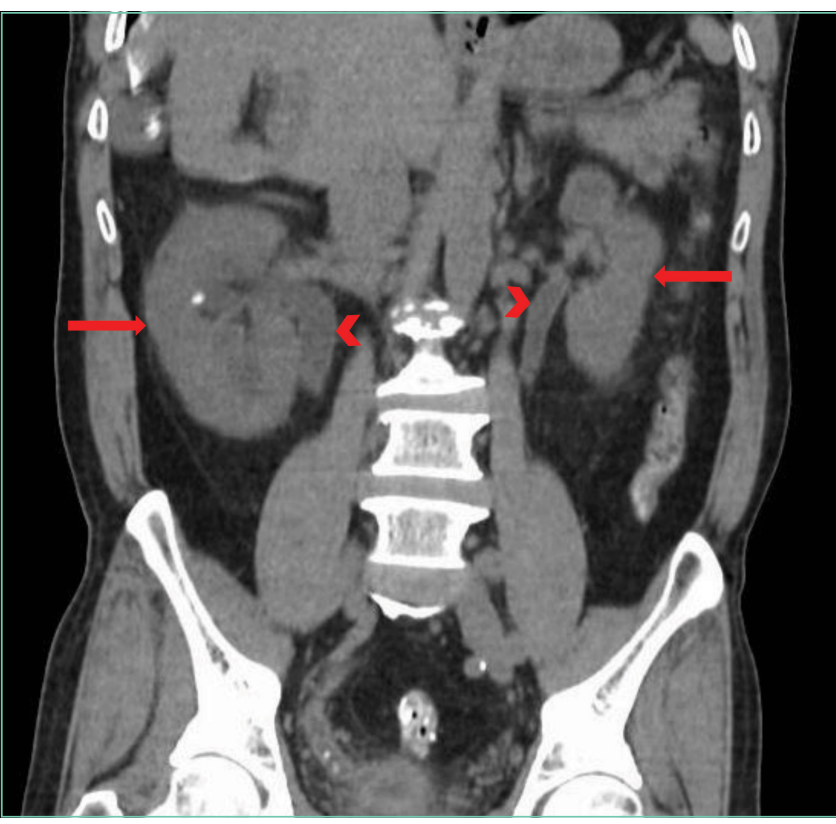

FIG 1. Coronal unenhanced abdominal CT scan shows (A) bilateral hydronephrosis (arrows) and (B) bilateral hydroureter (arrowheads).

mon causes include nephrolithiasis, urinary tract infection (UTI), prostatitis, neoplasm, and glomerulonephritis (GN). The lack of casts and dysmorphic RBCs makes GN unlikely. Taken together, his vital signs, examination, and laboratory studies suggest a high likelihood of an upper UTI (acute obstructive pyelonephritis) in the context of $\mathrm{AKI}$ due to obstructive uropathy.

Despite both a normal serum WBC count (which has only a moderate negative predictive value) and his low risk of developing life-threating organ dysfunction from sepsis based on a quick Sequential Organ Failure Assessment (qSOFA) score of zero, it is appropriate to start antibiotics after drawing blood and doing urine cultures. The next step should include administration of a broad-spectrum regimen that is appropriately dose-adjusted for renal dysfunction, such as an antipseudomonal carbapenem and vancomycin to cover extended-spectrum beta-lactamase (ESBL)-producing organisms, Pseudomonas aeruginosa, and methicillin-resistant Staphylococcus aureus (MRSA). This broad coverage is indicated for empiric treatment of complicated obstructive pyelonephritis, a condition that may arise from significant urinary obstruction and that carries a high risk of rapid clinical deterioration. He should undergo a rapid bedside test to assess for urethral or bladder outlet obstruction: either a bladder ultrasound or temporary insertion of a bladder catheter. He should also have an urgent computed tomography (CT) of his abdomen and pelvis without intravenous (IV) contrast, looking for evidence of urinary tract obstruction. A CT is preferred over ultrasound of the kidneys and bladder as CT has higher sensitivity and specificity for nephrolithiasis and neoplasm.

A CT of the abdomen and pelvis without IV contrast revealed bilateral hydroureter and hydronephrosis with multiple punctate calcified stones within the right calyces and the distal right ureter (Figure 1, Figure 2). However, these

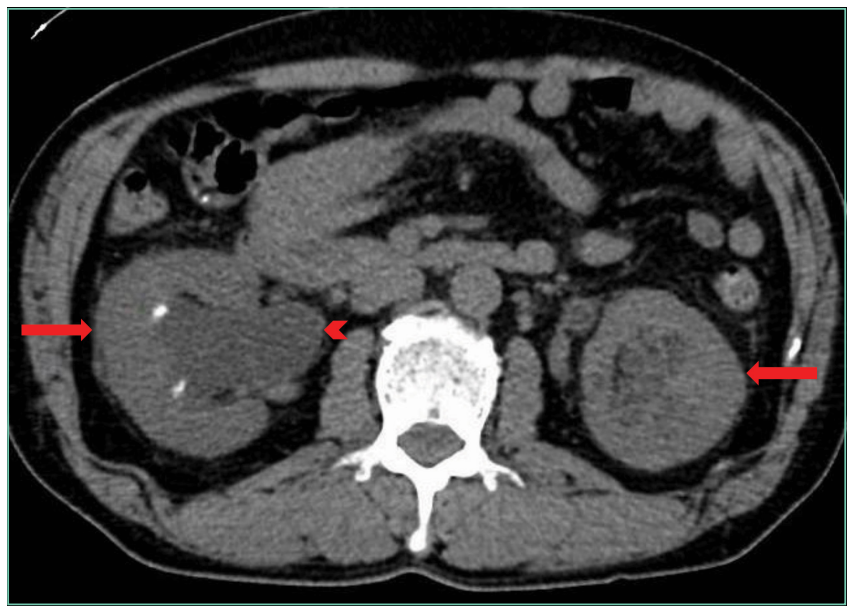

FIG 2. Axial unenhanced abdominal CT scan shows (A) bilateral hydronephrosis (arrows) and (B) more prominent hydroureter (arrowhead)

appeared too small to cause the degree of obstruction visualized. There were no stones noted in the left ureter to account for the obstruction, though small stones were noted in the left calyces. The bladder appeared normal.

Rarely are both ureters obstructed proximal to the ureterovesical junctions in the retroperitoneum. When they are, CT scans usually reveal culprit lesions that are extrinsic to the urinary tract, such as masses or retroperitoneal fibrosis, the latter of which can be associated with IgG4-related disease. Intrinsic causes of urinary tract obstructions include ureteral strictures (from infections, nephrolithiasis, instrumentation, prior radiotherapy, or rarely urothelial cancer), blood clots, metastatic ureteral deposits, or nephrolithiasis. While most intrinsic causes are unilateral, the patient is predisposed to strictures given his history of ureteral instrumentation. A preexisting unilateral obstruction due to a stricture may now, therefore, be unmasked by a second intrinsic obstruction in the contralateral ureter. Alternatively, given his remote history of living in the Philippines, a site where Schistosoma haematobium is endemic, chronic genitourinary schistosomiasis may have caused ureteral strictures due to granulomas, fibrosis, or pseudopolyps.

More commonly, bilateral hydroureter with bilateral hydronephrosis is caused by an obstruction of the bladder or urethra. CT scans can reveal prostatic hyperplasia (occasionally with protrusion into the bladder) and increased bladder wall thickness as a result of chronic bladder outlet obstruction, but the negative predictive value of either finding is modest. More revealing is that the patient reported neither an inability to pass urine (in fact, a "random" urine sample was obtained) nor suprapubic discomfort. Both symptoms would be pronounced with acute bladder obstruction but may be minimal with slowly progressive obstruction. In either case, a distended bladder would have been seen on the CT scan.

Regardless of the cause or whether the obstruction is in the upper or lower urinary tract, emergency intervention is needed to relieve the obstruction when AKI presents with bilateral hydronephrosis. A urology consultation should be sought ur- 
gently to determine the best strategy to relieve the obstruction. This may include bilateral percutaneous nephrostomy tubes given that the obstruction appears to be above the level of the bladder. Their findings will also direct additional diagnostic workup.

The patient received ceftriaxone and underwent cystoscopy, which revealed a stricture of the distal bulbar urethra. The ureters and bladder could not be completely visualized due to hematuria. The urethral stricture was dilated, and a Foley catheter was placed. In the operating room, the patient had a fever of $103^{\circ} \mathrm{F}$ and developed severe hypotension unresponsive to $3 \mathrm{~L}$ of intravenous normal saline. Norepinephrine infusion was initiated for refractory hypotension.

Except for transurethral prostate resections, endoscopic urologic procedures rarely lead to a stricture of any segment of the urethra, suggesting that the previous ureteral stent placement and retrieval were not causal. Longstanding Mycobacterium tuberculosis or Schistosoma haematobium infection occasionally causes urethral strictures presenting as bilateral hydronephrosis. The multiple punctate calcified "stones" demonstrated on CT may suggest either diagnosis if they were actually calcified granulomas.

Regardless of the cause, most patients with a urethral stricture have chronic lower urinary tract symptoms such as decreased stream and the feeling of incomplete bladder emptying. Since this patient does not report these symptoms, it is important to consider if the stricture might be merely incidental. The absence of pain is more telling than the absence of chronic or recurrent symptoms. Lack of pain argues strongly against a pure de novo acute obstruction because abrupt stretching of the renal capsules and the walls of the collecting system is usually painful. Slow stretching caused by a progressive stricture may mask the pain of a superimposed acute obstruction. A blood clot, for example, may have precipitated an acute-on-chronic obstruction upon lodging at the urethral stricture.

The worsening systemic response to the procedure may be due to increased intravesical pressure by dilation and cystoscopy, which may have caused subsequent backflow of bacteria from the renal parenchyma into the circulation (pyelorenal backflow). The broad-spectrum antibiotic regimen suggested above and IV crystalloid infusion should be continued with close hemodynamic monitoring.

Treatment for severe sepsis was initiated with empiric piperacillin-tazobactam, ceftriaxone was discontinued, and the patient was transferred to the intensive care unit (ICU). Norepinephrine was discontinued after 24 hours. Despite the indwelling Foley catheter, his kidney function worsened (creatinine increased to 10.5 over the next 36 hours, and he remained oliguric). Therefore, bilateral percutaneous nephrostomy tubes were placed to relieve the ongoing obstruction. In the ICU, he remained febrile, despite receiving piperacillin-tazobactam, through hospital day 7. Serial blood and urine cultures all remained negative. HIV testing was negative. His chest radiograph was unremarkable, and transthoracic echocardiogram was normal. His creatinine improved but plateaued at $2.5 \mathrm{mg} / \mathrm{dL}$ by day 7 .

Worsening renal function (alongside oliguria or anuria) despite a functioning Foley catheter suggests either intrinsic renal disease or bilateral ureteral obstructions. The initial attempt at relieving the obstruction with a Foley catheter did not take into consideration the bilateral ureteral strictures. As a result, soon thereafter, the insertion of percutaneous nephrostomy tubes was necessary. Given the severity of his illness, underlying obstructive uropathy, and persistent fever, suggesting an ongoing infection, one strategy would be to continue antibiotics with broader coverage than piperacillin-tazobactam. This approach may be reasonable, given the emergence of ESBL organisms and the possibility of MRSA due to instrumentation. However, it is important to note that only sterile pyuria has been identified to date, which raises the possibility of nonbacterial infections. Although chronic infection with Schistosoma haematobium can cause bilateral ureteral strictures, associated fever is limited to the acute phase of infection and not the chronic obstructive phase, unless there is a superimposed infection. Genitourinary Mycobacterium tuberculosis remains a likely possibility, regardless of the unrevealing chest radiograph. Urine nucleic acid amplification and acid-fast bacilli (AFB) smear and culture, the best initial diagnostic test, should be sent. Although less definitive, a tuberculin skin test and an interferon-gamma release assay should also be conducted. Histopathology of the ureters obtained by repeat cystoscopy may be diagnostic, but given the limited visualization during the last cystoscopy and the recent dilation of the urethra, this option should be kept in reserve for now.

Antibiotics were discontinued on day 7, but the patient continued to experience ongoing fever. Urine Histoplasma and serum cryptococcal antigens were negative. His urine AFB smear was $1+$ positive. Liver function tests revealed a total protein of $7.0 \mathrm{~g} / \mathrm{dL}$, albumin $3.0 \mathrm{~g} / \mathrm{dL}$, total bilirubin 1.2 $\mathrm{mg} / \mathrm{dL}$, direct bilirubin $0.3 \mathrm{mg} / \mathrm{dL}$, alkaline phosphatase 418 $\mathrm{U} / \mathrm{L}$, aspartate aminotransferase $65 \mathrm{U} / \mathrm{L}$, alanine aminotransferase $88 \mathrm{U} / \mathrm{L}$, gamma-glutamyltransferase (GGT) $609 \mathrm{U} / \mathrm{L}$ (normal, 3-60), and lactate dehydrogenase $284 \mathrm{U} / \mathrm{L}$ (normal, 85-210).

Acid-fast bacillus in the urine strongly suggests Mycobacterium tuberculosis (MTB) with several reports of likelihood ratios greater than 10. Nevertheless, confirmation is needed to rule out nontuberculous mycobacteria because of potential hepatotoxicity from treatment. Up to six urine samples should be sent for mycobacterium culture. However, false negative rates of up to $90 \%$ are reported, and final test results can take up to two months, so other methods of confirmation should be simultaneously sought. A nucleic acid amplification test of urine could rule in a pathogenic species within 24 hours. Alternatively, the probability of a nonpathogenic colonizing species would be negligible if a caseating granuloma was found. Bi- 


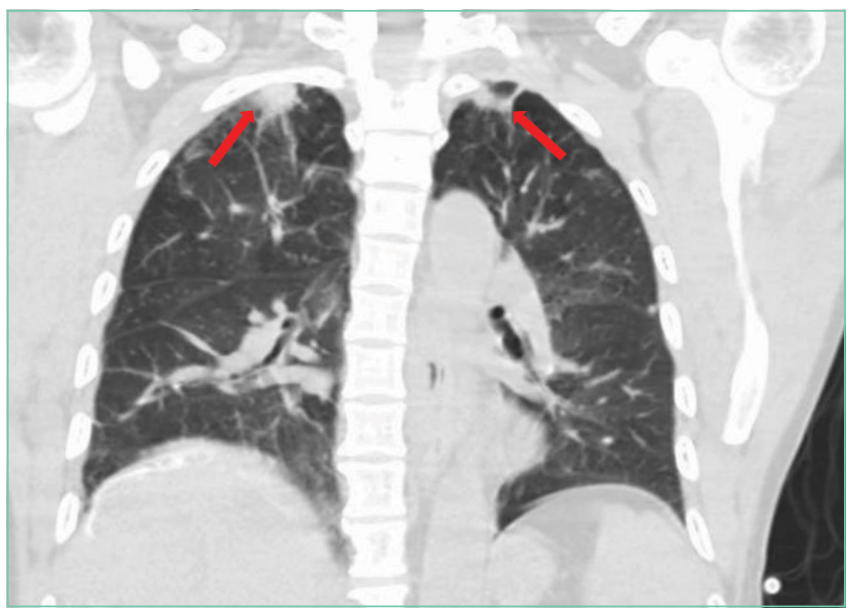

FIG 3. Coronal unenhanced chest CT scan shows bilateral fibrotic upper lobe opacities (arrows).

opsy could be obtained from the ureters, as suggested above. Liver biopsy should also be considered, especially if the moderate elevations in alkaline phosphatase and GGT (the most common liver enzyme abnormalities in hepatic tuberculosis) did not merely wax and wane with sepsis.

A CT of the thorax without IV contrast was done to evaluate for evidence of pulmonary disease given the positive urine AFB. This demonstrated bilateral fibrotic upper lobe opacities suggestive of prior granulomatous disease but no cavitary lung lesions (Figure 3). Three sputum smears were negative for AFB, but one sample showed Mycobacterium tuberculosis detected by a polymerase chain reaction (PCR) probe.

Given the concern for genitourinary tuberculosis (GUTB), it is appropriate to place the patient in respiratory isolation to exclude concomitant pulmonary tuberculosis (TB). AFB smears were negative, but the sputum PCR probe was positive, confirming pathogenic MTB. However, the negative AFB smears make the likelihood of pulmonary infectivity low. As a result, contact tracing is often deemed unnecessary by hospital infection control teams. Though his chest radiograph was normal, CT showed bilateral upper lobe fibrotic disease suggestive of prior pulmonary TB, thus making it likely that the current GUTB represents reactivation.

The two-month initiation phase of treatment with four antituberculosis drugs should begin while drug susceptibility tests are pending. Potential hepatotoxicity should be closely monitored, ideally by a clinician with experience treating tuberculosis in patients with existing liver disease. As a general precaution, alcohol should be avoided as should medications such as acetaminophen that are known to be hepatotoxic. Urology follow-up is also needed because about one-third of tuberculous ureteral strictures treated initially with percutaneous nephrostomy do not resolve with antituberculosis therapy.

The patient was started on weight-based antituberculosis treatment with four antimicrobial agents (rifampin, ethambutol, pyrazinamide, and isoniazid). He was seen in the in-

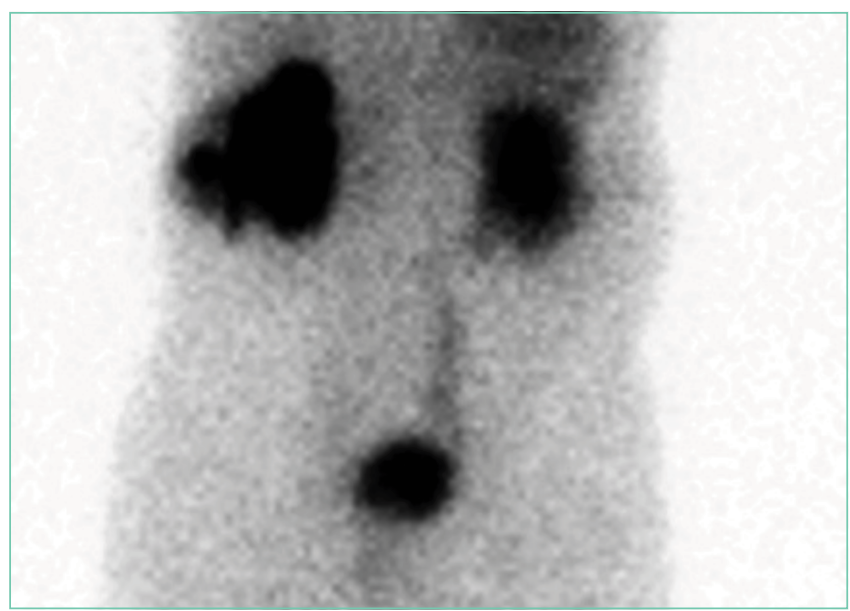

FIG 4. Renal scan showing decreased flow and function in the left kidney with scintigraphic evidence of outflow obstruction

fectious disease clinic two weeks later; his fever had resolved, and his liver function tests showed normalization of AST and $\mathrm{LDH}$ as well as a $45 \%$ reduction in his GGT and alkaline phosphatase levels. Two months following discharge, a nuclear medicine radionuclide angiogram renal flow scan showed normal right kidney function. The right nephrostomy tube was subsequently removed. He continued to have left kidney outflow obstruction due to a residual ureteral stricture (Figure 4). Repeat cystoscopy and attempted left ureteral stenting was unsuccessful. The left nephrostomy tube remained in place.

\section{DISCUSSION}

According to the Centers for Disease Control, in 2017, 10 million people became sick with $\mathrm{TB}$, and there were 1.3 million TB-related deaths worldwide with 9,150 cases reported in the United States. Extrapulmonary TB (EPTB) constitutes 10\% of all TB cases globally. ${ }^{1-4}$ GUTB is the second most common form of EPTB after lymph node TB, and it occurs in up to $20 \%$ of all pulmonary TB cases. ${ }^{2,3}$

Mycobacteria reach the genitourinary (GU) tract via hematogenous spread during primary infection or reactivation of TB. This leads to cortical and medullary lesions, which can heal spontaneously or eventually (average of 22 years) rupture into the tubules and onto the collecting system, ureters, and bladder. ${ }^{5,6}$ Spread to the ureter and bladder leads to multiple ureteral strictures and contracture of the bladder with disruption of the ureterovesical junction (UVJ), which causes hydroureter and hydronephrosis. ${ }^{7}$ Unilateral kidney involvement is most common, but bilateral involvement can occur following exacerbated hematogenous spread, particularly in immune deficient patients. Bilateral kidney involvement is also possible from retrograde spread to the good kidney due to bladder contracture and UVJ disruption. 8,9 Distal infection can involve all aspects of the male and female genital tracts, but urethral strictures are extremely rare..$^{10,11}$

GUTB affects males more than females (2:1) and presents insidiously at 40 to 60 years of age. ${ }^{12}$ Other risk factors for TB include birth in TB endemic areas, prior TB infection, immunosup- 
pression, malnutrition, severe systemic disease, diabetes, and cirrhosis. It is crucial to assess these risk factors when creating and refining differential diagnoses. Many patients have hematuria and sterile pyuria as incidental initial findings. The most common symptoms arise from bladder involvement, including frequency, urgency, and dysuria. Low back pain and gross hematuria are also common, but fever and constitutional symptoms are uncommon. ${ }^{10}$ Bilateral ureteral strictures can lead to obstructive renal failure, and involvement of the genital tracts can lead to pelvic or scrotal pain, swelling, and fistula formation. ${ }^{10}$

Diagnosis involves the demonstration of TB bacilli in urine or $\mathrm{GU}$ tissue. The urinalysis reveals hematuria and sterile pyuria. ${ }^{13}$ Urine AFB stains are positive in $52 \%$ of cases but are not diagnostic as nontuberculous mycobacteria may also cause a positive test result. 13,14 Urine cultures for Mycobacterium tuberculosis are positive in up to $90 \%$ of cases after six to eight weeks. As many as three to six morning urine samples are required to achieve diagnostic accuracy. ${ }^{10,14}$ Urine PCR for Mycobacterium tuberculosis has $96 \%$ sensitivity and up to $98 \%$ specificity, ${ }^{14}$ while PCR on GU tissue has a sensitivity of $88 \%$ and specificity of $87 \% .{ }^{15}$ The rapid nucleic acid amplification assay Xpert MTB/RIF in urine has a sensitivity of $83 \%$, and specificity of $98 \% .{ }^{16}$ Imaging is required to evaluate for obstruction, and the CT scan is abnormal in up to $90 \%$ of cases, showing multiple ureteral stenoses, hydroureter and hydronephrosis, and a contracted bladder. ${ }^{10,17}$

GUTB is treated with standard antituberculosis regimens. ${ }^{18}$ Patients with urinary obstruction benefit from ureteral stenting or percutaneous nephrostomy, bladder diversion, or ureteral reconstruction surgery. Unilateral nephrectomy for a nonfunctioning kidney with extensive disease is occasionally required. ${ }^{19}$ Following treatment, relapse occurs in up to $6 \%$ of patients over five years, and long-term follow-up with urine cultures and PCR every six months is recommended. ${ }^{10,20}$ Appropriate screening and treatment for latent tuberculosis infection greatly reduces the risk of reactivation GUTB.

This patient presented with features of an infection, which, combined with his history of renal stones and his urinalysis, led to an appropriate suspicion of and empiric treatment for an upper UTI. Given the AKI and nephrolithiasis, imaging was done to exclude obstruction. The CT finding of bilateral hydroureters and hydronephrosis absent an obstructing stone or mass or abnormal bladder was the initial clue that this was not a typical bacterial infection and that there was likely an underlying infectious pathologic process such as TB involving the GU tract diffusely. The care team treated the patient as an individual with fever and sterile pyuria in the context of multiple urinary tract strictures and an initial unrevealing infectious diagnostic workup. They recognized that the clues to the ultimate diagnosis of GUTB were all in the stream.

\section{KEY TEACHING POINTS}

- GUTB is a significant cause of sterile pyuria.

- In the presence of bilateral hydronephrosis, it is vital to determine the level of obstruction. If the bladder is not distended or contracted, then obstruction is likely at the level of the ureters and initial use of percutaneous nephrostomy tubes to relieve obstruction may be preferred.

- Imaging abnormalities such as multiple ureteral strictures, hydroureter and hydronephrosis (absent an obstructing stone or mass), and the finding of a contracted bladder are highly suggestive of GUTB.

- The mainstay of treatment for GUTB is standard antituberculosis treatment regimens in combination with the relief of urinary obstruction by ureteral stenting, percutaneous nephrostomy or open surgery.

- GUTB can relapse in up to $6 \%$ of treated cases over five years, and long-term follow-up and surveillance with urine culture and PCR every six months are recommended.

Disclosures: Benjamin Mba, Nathan Houchens, Marie Jennifer Seares, and Udit Joshi have no financial conflicts of interest and no disclosures.

Funding: Brian P. Lucas receives funding from the Department of Veterans Affairs, Veterans Health Administration, Office of Research and Development and Dartmouth SYNERGY, National Institutes of Health, and National Center for Translational Science (UL1TR001086).

\section{References}

1. Forssbohm M, Zwahlen M, Loddenkemper R, Rieder HL. Demographic characteristics of patients with extrapulmonary tuberculosis in Germany. Eur Resp J. 2008:31(1):99-105. https://doi.org/10.1183/09031936.00020607.

2. French CE, Antoine D, Gelb D, Jones JA, Gilbert RL, Watson JM. Tuberculosis in non-UK-born persons, England and Wales, 2001-2003. Int J Tuberc Lung Dis. 2007;11(5):577-584.

3. Peto HM, Pratt $\mathrm{RH}$, Harrington TA, LoBue PA, Armstrong LR. Epidemiology of extrapulmonary tuberculosis in the United States, 1993-2006. Clin Infect Dis. 2009;49(9):1350-1357. https://doi.org/10.1086/605559.

4. Alvarez S, McCabe WR. Extrapulmonary tuberculosis revisited: a review of experience at Boston City and other hospitals. Medicine. 1984;63(1):25-55

5. Simon HB, Weinstein AJ, Pasternak MS, Swartz MN, Kunz LJ. Genitourinary tuberculosis. Clinical features in a general hospital population. Am J Med. 1977;63(3):410-420. https://doi.org/10.1016/0002-9343(77)90279-0.

6. Christensen WI. Genitourinary tuberculosis: review of 102 cases. Medicine. 1974:53(5):377-390. https://doi.org/10.1016/0002-9343(77)90279-0.

7. Eastwood JB, Corbishley CM, Grange JM. Tuberculosis and the kidney. J Am Soc Nephrol. 2001;12(6):1307-1314.

8. Garcia-Rodriguez JA, Garcia Sanchez JE, Munoz Bellido JL, et al. Genitourinary tuberculosis in Spain: review of 81 cases. Clin Infect Dis.1994;18(4):557561. https://doi org/10.1093/clinids/18.4.557.

9. de Figueiredo AA, Lucon AM, Srougi M. Bladder augmentation for the treatment of chronic tuberculous cystitis. Clinical and urodynamic evaluation of 25 patients after long term follow-up. Neurourol Urodyn. 2006;25(5):433-440. https://doi.org/10.1002/nau.20264.

10. Figueiredo AA, Lucon AM, Srougi M. Urogenital Tuberculosis. Microbiol Spectr. 2017;5. https://doi.org/10.1128/microbiolspec.TNMI7-0015-2016.

11. Gupta N, Mandal AK, Singh SK. Tuberculosis of the prostate and urethra: A review. Indian J Urol. 2008;24(3):388-391. https://doi.org/10.4103/09701591.42623.

12. Figueiredo AA, Lucon AM, Junior RF, Srougi M. Epidemiology of urogenital tuberculosis worldwide. Int J Urol. 2008;15(9):827-832. https://doi. org/10.1111/j.1442-2042.2008.02099.x

13. Mortier E, Pouchot J, Girard L, Boussougant $Y$ Vinceneux P. Assessment of urine analysis for the diagnosis of tuberculosis. BMJ (Clinical research ed). 1996;312:27-28. https://doi.org/10.1136/bmj.312.7022.27

14. Moussa OM, Eraky I, El-Far MA, et al. Rapid diagnosis of genitourinary tuberculosis by polymerase chain reaction and non-radioactive DNA hybridization. J Urol. 2000;164(2):584-588. https://doi.org/10.1016/S0022-5347(05)67427-7.

15. Chawla A, Chawla K, Reddy S, et al. Can tissue PCR augment the diagnostic accuracy in genitourinary tract tuberculosis? Urol Int. 2012;88(1):34-38. https://doi.org/10.1159/000327039.

16. Kohli M, Schiller I, Dendukuri N, et al. Xpert((R)) MTB/RIF assay for extrapulmonary tuberculosis and rifampicin resistance. Cochrane Database Syst Rev. 2018;8:Cd012768. https://doi.org/10.1002/14651858.CD012768.pub2.

17. Figueiredo AA, Lucon AM, Arvellos AN, et al. A better understanding of urogenital tuberculosis pathophysiology based on radiological findings. Eur J Radiol. 2010;76(2):246-257. https://doi.org/10.1016/j.ejrad.2009.05.049.

18. Treatment of Tuberculosis: Guidelines. 4th edition. Geneva: World Health Organization. 2010

19. O'Flynn D. Surgical treatment of genito-urinary tuberculosis. A report on 762 cases. $\mathrm{Br} J$ Urol. 1970;42(6):667-671. https://doi.org/10.1111/j.1464410X.1970.tb06789x

20. Butler MR, O'Flynn JD. Reactivation of genito-urinary tuberculosis: a retrospective review of 838 cases. Eur Urol. 1975;1:14-17. https://doi. org/10.1159/000455566 